\title{
Videothoracoscopy in pleural effusions: A review of 41 cases
}

\author{
Miktat Arif Haberal $^{a}$, Kerim Tülüce ${ }^{\mathrm{a}}$, Ersin Köksal ${ }^{\mathrm{b} *}$, Songül Özyurtª, Vaner Köksal ${ }^{\mathrm{c}}$, Zeynep Şentürk ${ }^{\mathrm{d}}$, Murat Civan ${ }^{\mathrm{e}}$ \\ ${ }^{a}$ Department of Thoracic Surgery, Rize State Hospital, Rize, Turkey \\ ${ }^{b}$ Department of Anesthesiology and Reanimation, Faculty of Medicine, Ondokuz Mayls University, Samsun, Turkey \\ ${ }^{c}$ Department of Neurosurgery, Faculty of Medicine, Recep Tayyip Erdoğan University, Rize, Turkey \\ ${ }^{d}$ Department of Microbiology, Gazi State Hospital, Samsun, Turkey \\ ${ }^{e}$ Department of Cardiology, Rize State Hospital, Rize, Turkey
}

\section{ARTICLE INFO}

\section{Article History}

Received

$19 / 09 / 2012$

Accepted

$11 / 01 / 2013$

\section{* Correspondence to:}

Ersin Köksal

Department of Anesthesiology and

Reanimation,

Ondokuz Mayıs University,

Samsun, Turkey

e-mail: ersin.koksal@omu.edu.tr

\section{Keywords:}

Pleural effusion

Pleurodesis

Retrospective

VATS

\author{
ABSTRACT
}

This study aimed to evaluate the diagnostic and therapeutic value of videothoracoscopy in patients with pleural effusion. A total of 41 patients underwent video-assisted thoracic surgery (VATS) were analyzed, 25 male and 16 female. The mean age was 52.1 years (ranged between 18 and 92 years). Pleural effusion was left sided in 19 cases (46.4\%), right sided in 14 cases (34.1\%) and bilateral in eight cases (19.5\%). Subjects with exudative and hemorrhagic pleural effusions in thoracentesis were included in the study. In all cases; complete blood count, electrocardiography (ECG), arterial blood gas, coagulation profile and respiratory function test (RFT) were performed. Two view chest $\mathrm{X}$-ray and computed tomography scans were done to determine the localization of fluid and to decide the access point to thorax prior to procedure. One-lung ventilation was achieved in 20 patients under general anestesia, whereas double lung ventilation was preferred in 13 cases intolerant of one-lung ventilation. The procedure was carried out under local anesthesia in eight cases due to their high risk for general anesthesia. An approximately $50 \mathrm{cc}$ of pleural fluid and at least two pieces of pleural biopsy specimen for cytopathological examination were collected in all cases. Among the study population, 33 of cases were diagnosed with a benign disease and the remaining eight cases with a malign disease. Taking age ranges into account, non-specific pleuritis and tuberculosis pleuritis were most commonly diagnosed between 20 to 40 years of age whereas malign pathologies between 40-60 years of age. Diagnostic success of the procedure was found to be $97.6 \%$. Patients with malign effusion underwent chemical pleurodesis with talc powder. No complication developed in our cases. VATS seems to be an effective method in the diagnosis and treatment of patients with pleural effusion.

J. Exp. Clin. Med., 2013; 30:35-37

\section{Introduction}

The first application of thoracoscopy in human beings was carried out by Hans Christian Jacobaeus with a cystoscope. In 1930, Dr. Anton Stattler defined the morphology of spontaneous pneumothorax, thoracoscopically and used talc powder method for its treatment (Panadero et al., 2006; Panadero, 2008). Video-assisted thoracic surgery (VATS) is widely used for the purpose of diagnosis and treatment by chest surgeons in current practice. The fields of its diagnostic usage include pleural effusions with unknown etiology, interstitial lung diseases, undefined lung nodules, mediastinal lymph node sampling and biopsies of mediastinal masses. VATS has also a wide range of treatment use such as ligation of bullae, thoracal sympathectomy, esophagial surgical interventions, mediastinal mass resection and pericardiectomy (Landreneau et al., 1994; Fowkers et al., 2009). The unique absolute contraindication of the procedure is the absence of pleural space. Relative contraindications include severe general health conditions of patients, hypoxemia independent of pleural effusion, fever, uncontrollable cough attacks, hemorrhagic diathesis and cardiological pathologies.

\section{Materials and methods}

The records of 41 patients with pleural effusion, who had 
been hospitalized in the Thoracic Surgery Department of Rize State Hospital between October 2005 and October 2011, were analyzed, retrospectively. Among the patients recruited for this study, 25 patients were male and 16 were female. The mean age was 52.1 years (ranged between 18 and 92 years). Preoperatively, all patients underwent thoracentesis and the effusion samples were subjected to cytological, biochemical and bacteriological studies. Cases with exudative and hemorrhagic pleural effusions in thoracentesis were included in the study. Patients other than eight cases were evaluated by diagnostic bronchoscopy. General anesthesia was employed in 33 cases, while the procedures of eight cases were performed under local anesthesia. In case of local anesthesia, cutaneous and subcutaneous region, muscles, rib surfaces and parietal pleura were desensitized with approximately $20 \mathrm{cc}$ of marcaine and the procedure was carried out with a rigid thoracoscope and biopsy forceps through a $1 \mathrm{~cm}$ and a $2 \mathrm{~cm}$-incision, respectively.

\section{Results}

Postoperative pathological study of cases who underwent VATS due to idiopathic pleural effusion revealed chronic non-specific pleuritis in 21 patients $(51.2 \%)$, tuberculous pleuritis in $10(24.3 \%)$, hemothorax in two (4.8\%), mesothelioma in four $(9.7 \%)$, metastasis of adenocarcinoma in three $(7.3 \%)$ and metastasis of epidermoid carcinoma in one patient $(2.4 \%)$ (Table 1$)$.

\section{Table 1. Histopathological results of pleural effusions}

\begin{tabular}{lcc} 
Histopathological findings & $\begin{array}{c}\text { VATS } \\
\text { diagnosis }\end{array}$ & $\begin{array}{c}\text { Final } \\
\text { diagnosis }\end{array}$ \\
\hline Chronical non-spesific pleuritis & 21 & 20 \\
Tuberculosis pleuritis & 10 & 11 \\
Malign mesothelioma & 4 & 4 \\
Metastasis of epidermoid carcinoma & 1 & 1 \\
Metastasis of adenocarcinoma & 3 & 3 \\
Hemothorax & 2 & 2 \\
Total & 41 & 41 \\
\hline
\end{tabular}

Pulmonary decortication was employed after 85 days following surgery in two cases with sequestered lungs and chronic nonspecific pleuritis. One of them was reported as tuberculous pleuritis after decortication while the diagnosis of other patient remained. Our cases with a reported malign pathology and a patient with nonspecific pleuritis (pleural effusion secondary to chirrosis) were treated with chemical pleurodesis using talc powder. Anti-tuberculosis treatment was initiated in cases reported as tuberculous pleuritis after pathological examination. No pleural fluid accumulation or pleural thickening was detected in the follow-up records of these cases. Taking age ranges into account, non-specific

\begin{tabular}{lccccc}
\multicolumn{2}{l}{ Table 2. Age ranges of patients } & & & \\
Histopathological diagnosis & $\begin{array}{c}\mathbf{0 - 2 0} \\
\text { years }\end{array}$ & $\begin{array}{c}\mathbf{2 0 - 4 0} \\
\text { years }\end{array}$ & $\begin{array}{c}\mathbf{4 0 - 6 0} \\
\text { years }\end{array}$ & $\begin{array}{c}\mathbf{6 0 +} \\
\text { years }\end{array}$ \\
\hline Non-specific pleuritis & 4 & 11 & 3 & 2 \\
Tuberculous pleuritis & 4 & 5 & 1 & - \\
Malign mesothelioma & - & - & 3 & 1 \\
Metastasis of carcinoma & - & - & 2 & 2 \\
Hemothorax & 1 & 1 & - & - \\
\hline
\end{tabular}

Diagnostic success of VATS in younger patients diagnosed as nonspecific pleuritis was found to be $95.3 \%$. pleuritis and tuberculosis pleuritis were most commonly diagnosed between 20 to 40 years of age whereas malign pathologies between $40-60$ years of age (Table 2).

\section{Discussion}

Pleural effusion results from a disturbance in balance between secretion and absorbtion of pleural fluid. Increased pleural fluid production or decreased lymphatic absorbtion or both of these, simultaneously, may cause fluid accumulation in pleural cavity (Ergin et al., 2011). Pleural effusions may result from various types of diseases. Sometimes the cause of effusion may be so obvious due to its clinical presentation that there is no need to further investigate (e.g. bilateral pleural effusion in heart failure without any fever and pleuritic pain). Mostly, invasive diagnostic interventions are needed to sample pleural fluid and to reveal the underlying etiology. First step in diagnosis process is to begin with the least invasive procedure, namely thoracentesis which is performed in non-loculated effusions preferably through posterior side of $8^{\text {th }}$ or $9^{\text {th }}$ intercostal space. In loculated effusions, on the other hand, the procedure is carried out as ultrasound-guided. Thoracentesis and percutaneous pleural biopsy are associated with a lower diagnostic value. Etiologies of pleural effusions are revealed $40-87 \%$ with thoracentesis, $39-75 \%$ with percutaneous pleural biopsy and almost 100\% with VATS (Batirel and Yuksel, 2002). From 41 cases with pleural effusion or pleural thickening recruited into our study, four cases were reported as malign mesothelioma including one mixed, one sarcomatous and two epithelial subtypes. Metastatic malign effusions were caused by adenocarcinoma in three cases (7.3\%) and epidermoid carcinoma in one case $(2.4 \%)$. A patient $(2.4 \%)$ reported as non-specific pleuritis with VATS was diagnosed with tuberculous pleuritis after pulmonary decortication. Although diagnosis rate of pleural needle biopsy in tuberculous pleural effusions is $54-57 \%$, repeated biopsies and tissue cultures may raise it to $80-90 \%$ (Wrightson et al., 2009). Malign pleural effusion (MPE) develops secondary to pleural tumors and is the most common effusion encountered by thoracic surgeons. It stems typically from pleural invasions of lung and breast carcinoma, and less likely from invasions of lymphoma, ovarian and gastric carcinoma. This clinical presentation indicates a shorter life expectancy almost in all cases (Sahn, 2001). VATS is also widely used in the treatment of pleural diseases in addition to making the diagnosis (Kürkçüoğlu et al., 2000; Ahmed and Jones, 2004; Fabbrucci et al., 2008). Currently, its most common indications are pleural effusions with unknown etiologies, excision of bullae and thoracic symphatectomy. Absolute contraindications of thoracoscopy include inadequate space in pleural cavity, diffuse adhesions, patients in comatose or unconscious state, respiratory insufficiency, severe pulmonary hypertension and end stage pulmonary fibrosis. Its relative contraindications are severe cough attacks, bleeding diathesis, thrombocytopenia, decompensated heart failure, hypoxemia resistant to $\mathrm{O}_{2}$ treatment, thrombolysis history in last three months and fever (Artvinli, 2002; Ergin et al, 2011). Boutin et al. (1990) reported a diagnosis rate of $59 \%$ in their 1000 cases Kürkçüoğlu et al. (2000) also declared that they reached a diagnosis with a success rate of $96 \%$ via thoracoscopy in a case series of 215 patients. In our two cases with history of thoracic trauma, hemorrhagic effusion was aspirated and no accompanying 
pathology was detected. One of our cases with massive effusion secondary to liver cirrhosis underwent VATS, thereby the effusion was aspirated, pleural surfaces were evaluated and a chemical pleurodesis with 4 gram talc powder was carried out. To avoid recurrent effusions, chemical pleurodesis was employed in all cases with malign diseases using 4 grams of talc powder through chest tubes following the expansion of lungs and a tube drainage less than $100 \mathrm{cc}$. In cases with MPE, antineoplastic agents such as bleomycin and mitomy- cin, some antibiotics such as tetracyclin and doxicyclin, and interleukin-2 are used as chemical agents for pleurodesis (Kaifi et al., 2012). In our case series, we preferred talc powder which is cheap and provides a potent adhesive effect.

\section{Conclusion}

As a result of our analysis we suggest that VATS is a minimal invasive method useful in both diagnosis and treatment of pleural disease.

\section{REFERENCES}

Ahmed, N., Jones, D., 2004. Video-assisted thoracic surgery: State of the art in trauma care. Injury. 35, 479-489.

Artvinli, M., 2002. Classic thoracoscopy. Hacettepe Med. J. 33, 73-77.

Batirel, H.F., Yuksel, M., 2002. Evulation of pleural effusion: A Surgical Approach. Turk. Thorac. J. 3, 13-19.

Boutin, C., Astroul, P., Seitz, B., 1990. The role of thoracoscopy in the evaluation and management of pleural effusion. Lung. 168, $1113-1121$. Ergin, M., Gürlek, K., Yeginsu, A., Ergin, İ., 2011. Invasive approaches to the diagnosis of pleural effusion. J. Clin. Anal. Med. 2, $43-46$.

Fabbrucci, P., Nocentini, L., Secci, S., Manzoli, D., Bruscino, A., Fedi, M., Paroli, G.M., Santoni, S., 2008. Video-assisted thoracoscopy in the early diagnosis and management of post traumatic pneumothorax and hemothorax. Surg. Endosc. 22, 1227-1231.

Fowkers, L., Lau, K.K.W., Shan, N.,Black, E., 2009. A cervical approach to investigating pleural disease. Ann. Thorac. Surg. 88, $315-317$.

Kaifi, J.T., Toth, J.W., Gusani, N.J., Kimchi, E.T., Staveley-O'Carroll, K.F., Belani, C.P., Reed, M.F., 2012. Multidisciplinary management of malignant pleural effusion. J. Surg. Oncol. 105, 731-738.

Kürkçüoğlu, C., Karaoğlanoğlu, N., Eroğlu, A., Ünlü, M., 2000. Pleural effusions and video assisted thoracoscopy. J. Turk. Thorac. Cardiovasc. Surg. 8, 712-714.

Landreneau, R.J., Hazelrigg, S.R., Mack, M.J., 1994. Video-assisted thoracic surgery for pulmonary and pleural diseases. In: Shields TW, ed. General Thoracic Surgery. Malvern: Williams\&Wilkins. 508-526.

Panadero, F.R., Janssen, J.P., Astoul, P., 2006. Thoracoscopy: General overview and place in the diagnosis and management of pleural effusion. Eur. Respir. J. 28, 409-421.

Panadero, F.R., 2008. Rigid thoracoscopy. TTD Plevra Bülteni. 2, 63-66.

Sahn, S.A., 2001. Management of malignant pleural effusions. Monaldi. Arch. Chest. Dis. 56, 394-399.

Wrightson, J.M., Helm, E.J., Rahman, N.M., Gleeson, F.V., Davies, R.J.O., 2009. Pleural procedures and pleuroscopy. Respirology. 14, 796807. 